\title{
Hazard-evaluation-oriented Moving Horizon Parallel Steering Control for Driver-Automation Collaboration during Automated Driving
}

\author{
Hongyan Guo, Member, IEEE, Linhuan Song, Jun Liu, Fei-Yue Wang, Fellow, IEEE, Dongpu Cao, Member, IEEE, \\ Hong Chen, Senior Member, IEEE, Chen Lv, Member, IEEE, Partick Chi-Kwong Luk, Senior Member, IEEE,
}

\begin{abstract}
Prompted by emerging developments in connected and automated vehicles, parallel steering control, one aspect of parallel driving, has become highly important for intelligent vehicles for easing the burden on and ensuring the safety of human drivers. This paper presents a parallel steering control framework for an intelligent vehicle using moving horizon optimization. The framework considers lateral stability, collision avoidance and actuator saturation and describes them as constraints, which can blend the operation of a human driver and a parallel steering controller effectively. Moreover, the road hazard and the steering operation error are employed to evaluate the operational hazardous of an intelligent vehicle. Under the hazard evaluation, the intelligent vehicle will be mainly operated by the human driver when the vehicle operates in a safe and stable manner. The automated steering driving objective will play an active role and regulate the steering operations of the intelligent vehicle based on the hazard evaluation. To verify the effectiveness of the proposed hazard-evaluation-oriented moving horizon parallel steering control approach, various validations are conducted, and the results are compared with a parallel steering scheme that does not consider automated driving situations. The results illustrate that the proposed parallel steering controller achieves acceptable performance under both conventional conditions and hazardous conditions.
\end{abstract}

Index Terms-Parallel steering control, Moving horizon optimization, Hazard evaluation, Intelligent vehicle, Lateral stability

\section{INTRODUCTION}

This work is supported by the National Nature Science Foundation of China (61520106008, 61790563, U1664263).(Corresponding author: Hong Chen and Dongpu Cao)

H. Guo, L. Song, J. Liu, and H. Chen are with the State Key Laboratory of Automotive Simulation and Control and the Department of Control Science and Engineering, Jilin University (Campus NanLing), Changchun 130025, PR China (e-mail: guohy11@jlu.edu.cn; liujun16@mails.jlu.edu.cn; chenh@jlu.edu.cn).

F.-Y. Wang is with the State Key Laboratory of Management and Control for Complex Systems. Institute of Automation, Chinese Academy of Sciences, China, and also with the Research Center for Military Computational Experiments and Parallel Systems Technology, National University of Defense Technology, Changsha 410073, China (e-mail: feiyue.wang@ia.ac.cn).

D. Cao is with the Mechanical and Mechatronics Engineering Department at the University of Waterloo, 200 University Avenue West Waterloo, ON, N2L 3G1, Canada(e-mail: dongp_ca@yahoo.com).

C. Lv are with the Department of Automotive Engineer, Cranfield University, Bedfordshire MK43 0AL, UK (e-mail: d.cao@cranfield.ac.uk; c.lyu@ cranfield.ac.uk).

P. C. K. Luk is with the School of Water, Energy and Environment, Cranfield University, Bedfordshire MK43 0AL, UK (e-mail: p.c.k.luk@cranfield.ac.uk).
W ITH the rapid development of parallel control and management theory and its numerous applications in transport automation and vehicle intelligence over the past decade [1], [2], parallel steering control, which is an aspect of parallel driving, has been steadily developed and applied in practice [3], [4]. Moreover, furthered by emerging developments in connected and automated vehicles [5], [6], parallel steering control has become a hot topic and has been garnering increased attention from both academic and industrial researchers [7].

Parallel steering control systems are composed of three components: an intelligent vehicle, a human driver and a parallel steering controller. The steering operation of the human driver and the computed steering wheel angle by the parallel steering controller are coupled by the intelligent vehicle [8], [9]. To ensure that an intelligent vehicle runs in a safe and stable manner, the final steering operation should be compared and evaluated between the human driver and the parallel steering controller, therein employing vehicle dynamic states and traffic conditions [10]. Due to its online optimization, preview performance and constraint-handling capabilities, model predictive control (MPC) represents an opportunity to provide parallel steering control for an intelligent vehicle while satisfying safety constraints [11].

Among many of the studies related to the parallel steering control issue, several schemes can be classified based on the stage of the research. One type of approach simply guides a driver that does not actively control the vehicle. In [12], a MPC approach was employed to generate optimal paths to help guide a human driver using information about the surrounding environment. In [13], a co-pilot driving scheme was expanded and improved, and an automated driving capability is presented. In these approaches, the vehicle is always controlled by the human driver. The parallel steering controller only provides driving advice. The controller cannot effectively avoid traffic in situations of improper operation, driver distraction or driver inattentiveness.

Another approach is to directly switch control between a human driver and a parallel steering controller. In [14], a switching strategy was presented to govern the driver-assistance interaction by describing the hybrid system as an input-output hybrid automation. In this situation, the judgment-and-switch strategy is conducted by analyzing the state of the human driver and both traffic and road conditions. However, the stability at the switching point needs to be further discussed 
in this scheme.

In contrast to the above-mentioned switch control, the interpretation of the driver's driving intention is adopted and described as a reference path, and a controller is designed to follow the path. In [15], a lane-keeping strategy was designed to share control of the steering wheel with the driver in an optimal manner by describing the problem as an $\mathrm{H}_{2}$-preview optimization control issue. Because the proposed scheme follows a given or predefined path based on an interpretation of the driver's intention, the rationality and consistency with the driver should be further discussed.

In addition to the above-mentioned schemes, there is a parallel steering control approach that issues a final steering command by blending human driver steering operation and an optimal controller. In [16], a shared control framework for obstacle avoidance and stability control was presented using an MPC approach. The final steering command was a blended value decided by a human driver and a parallel controller simultaneously. The objective of this approach mainly follows the operation of a human driver. This will be hazardous when a human driver improperly operates the vehicle or is under certain driving conditions whereby an intelligent vehicle cannot be controlled by the human driver.

In this manuscript, motivated by the shared control scheme in [16], a parallel steering control framework is developed by blending the operation of a human driver and a parallel steering controller using a moving horizon optimization approach. The path following error and the steering operation error are employed to evaluate the current hazardous situation of the intelligent vehicle. Under the hazard evaluation, the intelligent vehicle will be mainly operated by the human driver when the vehicle runs in a safe and stable manner. In addition, the automated steering driving objective will play an active role and regulate the steering operation applied to the intelligent vehicle based on the hazard evaluation. Moreover, lateral stability, collision avoidance and actuator saturation are considered by the parallel steering controller. To verify the effectiveness of the proposed hazard-evaluation-oriented moving horizon parallel steering control approach, various validations are conducted and compared with a parallel steering scheme not considering the automated driving situation.

The two main contributions of this manuscript are as follows: (1) a parallel steering control framework is developed using a moving horizon optimization whereby the operation by the human driver is dominant during safe operating conditions but whereby the automated steering driving objective will regulate the steering operation in hazardous situations. (2) The hazard evaluation is performed for the parallel steering control whereby the road hazard and the steering operation error are employed to evaluate the current hazardous situation of the intelligent vehicle.

The remainder of this paper is organized as follows: In Section II, the intelligent vehicle model and the problem description are presented. In Section III, the parallel steering control framework using moving horizon optimization is presented, and the hazard evaluation is discussed. In Section IV, a highprecision verification vehicle model for is built, and then, joint veDYNA-Simulink simulations are performed under various driving conditions. Finally, in Section V, the conclusions are presented.

\section{Vehicle Model AND PROBlem Description}

\section{A. Vehicle Model}

A human driver makes decisions according to traffic conditions and a vehicle's position and dynamic states. Therefore, the vehicle model used in parallel steering control should also describe the vehicle position and lateral dynamics.

Under the assumption that the intelligent vehicle is a rigid body with non-deformable wheels, the longitudinal and lateral positions as well as the yaw angle of the vehicle can be described according to the geometric relationship described in Fig. 1 (a):

$$
\begin{aligned}
& \dot{x}_{o}=v \cos (\psi+\beta), \\
& \dot{y}_{o}=v \sin (\psi+\beta), \\
& \dot{\psi}=r,
\end{aligned}
$$

where $x_{o}$ and $y_{o}$ are the longitudinal and lateral positions of

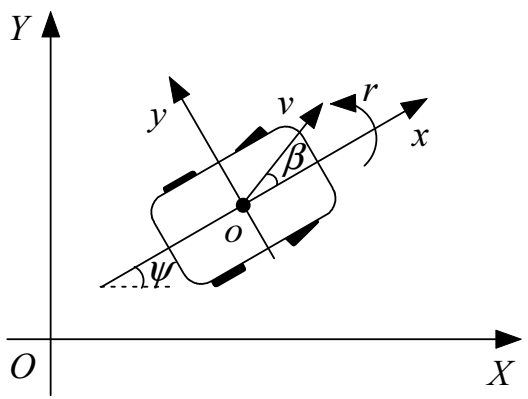

(a) Vehicle kinematic relation

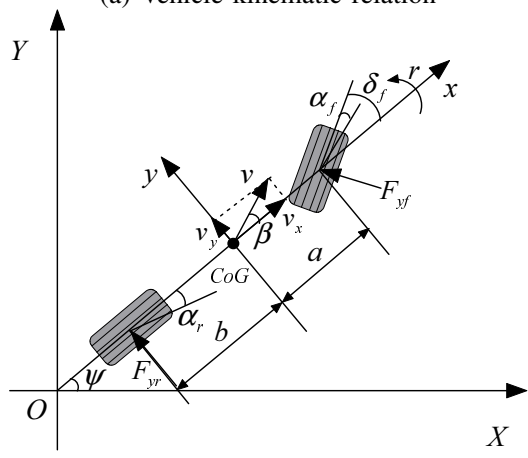

(b) 2-DOF vehicle model

Fig. 1. Vehicle model

the center of gravity (CoG), $r$ is the yaw rate, $\beta$ is the vehicle sideslip angle, $\psi$ is the yaw angle, and $v$ is the longitudinal velocity of the CoG. Considering that the variances of the yaw angle and sideslip angle are small and that the road curvature is also small, it can be assumed that $\sin (\psi+\beta) \approx \psi+\beta$ and $\cos (\psi+\beta) \approx 1$. Then, the simplification can be made:

$$
\begin{aligned}
& \dot{x}_{o}=v, \\
& \dot{y}_{o}=v(\psi+\beta), \\
& \dot{\psi}=r .
\end{aligned}
$$

Considering that the lateral dynamics of the intelligent vehicle is also needed in the parallel steering control, the 
vehicle body coordinate system with the origin at the $\mathrm{CoG}$ is defined as shown in Fig. 1 (b), where the positive direction of the $x$-axis points in the forward direction, the positive direction of the $y$-axis points to the left, and the directions of the $z$-axis and other forces and torques are determined by the right-hand rule. Assuming that the longitudinal velocity is constant, the lateral dynamics of the vehicle can be derived as follows:

$$
\begin{aligned}
m v(\dot{\beta}+r) & =F_{x f} \sin \delta_{f}+F_{y f} \cos \delta_{f}+F_{y r}, \\
I_{z} \dot{r} & =a\left(F_{x f} \sin \delta_{f}+F_{y f} \cos \delta_{f}\right)-b F_{y r},
\end{aligned}
$$

where $m$ is the vehicle mass; $I_{z}$ is the moment of inertia of the vehicle about the $z$-axis; $F_{x f}$ is the longitudinal tire-road force; $a$ and $b$ are the distances from the $\mathrm{CoG}$ to the front and rear axles, respectively; $F_{y f}$ and $F_{y r}$ are the front and rear lateral tire forces, respectively; and $\delta_{f}$ is the front wheel steering angle. Using a small angle for the front steering wheel angle $\delta_{f}$, Eq. (3) is simplified as follows:

$$
\begin{aligned}
m v(\dot{\beta}+r) & =F_{y f}+F_{y r} \\
I_{z} \dot{r} & =a F_{y f}-b F_{y r}
\end{aligned}
$$

The lateral tire forces $F_{y f}$ and $F_{y r}$ can be obtained employing tire sideslip angles:

$$
\begin{aligned}
& F_{y f}=C_{f} \alpha_{f}, \\
& F_{y r}=C_{r} \alpha_{r},
\end{aligned}
$$

where $C_{f}$ and $C_{r}$ are the cornering stiffness of the tire. In addition, the front and rear tire sideslip angles of the vehicle $\alpha_{f}$ and $\alpha_{r}$ can be approximate as follows [17]:

$$
\begin{aligned}
& \alpha_{f}=\beta+\frac{a r}{v}-\delta_{f}, \\
& \alpha_{r}=\beta-\frac{b r}{v} .
\end{aligned}
$$

Then, substituting Eq. (5) and Eq. (6) into Eq. (4) and combining with Eq. (2), the lateral dynamics of an intelligent vehicle can be obtained as follows:

$$
\begin{aligned}
& \dot{y}_{o}=v(\psi+\beta), \\
& \dot{\psi}=r, \\
& \dot{\beta}=\frac{\left(C_{f}+C_{r}\right)}{m v} \beta+\left(\frac{\left(a C_{f}-b C_{r}\right)}{m v^{2}}-1\right) r-\frac{C_{f}}{m v} \delta_{f}, \\
& \dot{r}=\frac{\left(a C_{f}-b C_{r}\right)}{I_{z}} \beta+\frac{\left(a^{2} C_{f}+b^{2} C_{r}\right)}{I_{z} v} r-\frac{a C_{f}}{I_{z}} \delta_{f} .
\end{aligned}
$$

The steering system is assumed to be designed such that there is a proportional relationship between the steering wheel angle and the front wheel steering angle. Accordingly, given the relationship between the front wheel steering angle $\delta_{f}$ and the steering wheel angle $\delta_{s w}$, the steering wheel angle can be expressed as $\delta_{s w}=I_{s} \delta_{f}$, where $I_{s}$ is the steering ratio. Specifically, the front steering wheel angle can be transformed into the steering wheel angle. Therefore, the front steering wheel angle is employed here in the discussion of the parallel steering control in this manuscript. Then, selecting the lateral position $y_{o}$ as the output, the front wheel steering angle $\delta_{f}$ as input, and $x=\left[\begin{array}{llll}y_{o} & \psi & \beta & r\end{array}\right]$ as the states, the system shown in Eq. (7) can be described as follows:

$$
\begin{aligned}
& \dot{x}=A x+B \delta_{f}, \\
& y=C x,
\end{aligned}
$$

where

$$
\boldsymbol{A}=\left[\begin{array}{cccc}
0 & v & v & 0 \\
0 & 0 & 0 & 1 \\
0 & 0 & \frac{C_{f}+C_{r}}{m v} & \frac{a C_{f}-b C_{r}}{m^{m} v^{2}}-1 \\
0 & 0 & \frac{a C_{f}-b C_{r}}{I_{z}} & \frac{a^{2} C_{f}+b^{2} C_{r}}{I_{z} v}
\end{array}\right], \boldsymbol{B}=\left[\begin{array}{c}
0 \\
0 \\
-\frac{C_{f}}{m v} \\
-\frac{a C_{f}}{I_{z}}
\end{array}\right], \boldsymbol{C}=\left[\begin{array}{l}
1 \\
0 \\
0 \\
0
\end{array}\right]^{\mathrm{T}} .
$$

The linear single-track model is employed here to describe the dynamics of an intelligent vehicle and the parameters can be obtained in [6]. When the intelligent vehicle runs into the nonlinear region, the modelling error will become large. However, the objective of parallel steering control is ensuring the intelligent vehicle run in the linear region, which can be satisfied by the constraints of MPC optimization. Therefore, the linear single-track model is enough to discuss the parallel steering control here. To verify the precision of the proposed vehicle model, a comparison with the measured behavior of a test vehicle and the simulated output of a high-precision vehicle model implemented in veDYNA is performed, and the validation results are shown in Fig. 2. Moreover, it can be seen from Fig. 2 that the precision of the intelligent vehicle model is sufficient to address the parallel steering control problem. Then, by discretizing Eq. (8) at the sample time $T_{s}$ using a zero-order hold method, the discrete-time model can consequently be obtained as follows:

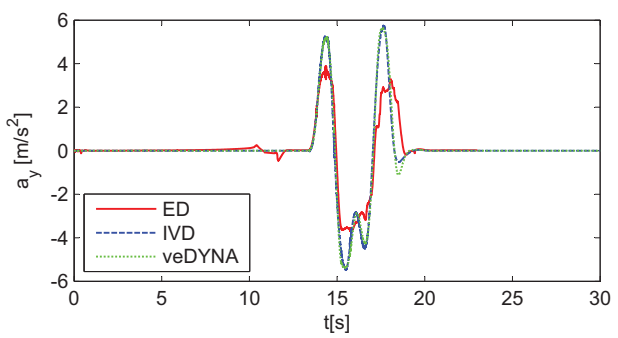

(b) lateral acceleration validation

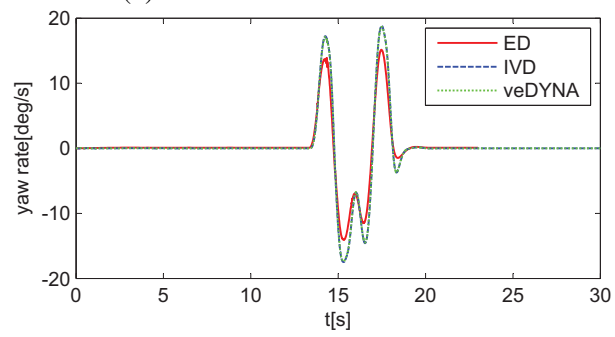

(c) yaw rate validation

Fig. 2. Model validation results for a standard double-lane-change maneuver with $v_{x}=60 \mathrm{~km} / \mathrm{h}$; "ED" denotes experimental data, "IVD" denotes the intelligent vehicle model presented here, and "veDYNA" denotes the vehicle model implemented in the veDYNA software

$$
\begin{aligned}
& x(k+1)=A_{d} x(k)+B_{d} \delta_{f}(k), \\
& y(k)=C_{d} x(k),
\end{aligned}
$$

where $A_{d}=e^{A T_{s}}, B_{d}=\int_{0}^{T_{s}} e^{A \tau} d \tau \cdot B, C_{d}=C$ are the discrete matrices.

\section{B. Problem Description}

Considering the intelligent vehicle operating on the road shown in Fig. 3 with a constant longitudinal velocity, the steering operation that is integrally decided upon by the human 
driver and autonomous driving system should be performed to follow the desired path. The steering operation by the human driver is accepted and kept invariant when the vehicle operates safely in this situation. In contrast, if the human driver steers the intelligent vehicle too far from the desired path or makes the intelligent vehicle unstable, the parallel steering control system corrects the steering operation of the human driver until the intelligent vehicle follows the desired road safely.

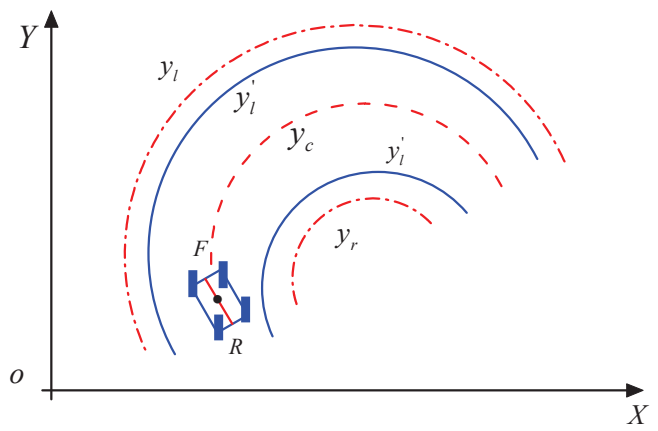

Fig. 3. Schematic diagram of the intelligent vehicle operating on the road

In the above-mentioned parallel steering control framework, the road shown in Fig. 3 is represented by three curves: the centerline of the road, $y_{c}$; the left road boundary, $y_{l}$; and the right road boundary, $y_{r}$. Moreover, the road centerline can be computed as $y_{c}=\frac{1}{2}\left(y_{l}+y_{r}\right)$. It is assumed that the left and right road boundaries can be obtained by on-board cameras. The shape of the intelligent vehicle operating on the road is described as a rigid bar with a length of $l$. Because the width of the intelligent vehicle is not considered, each boundary of the feasible road region is reduced by a half of the width of the intelligent vehicle to ensure the rationality of the description of the intelligent vehicle, that is, $y_{l}^{\prime}=y_{l}-\frac{w}{2}$ and $y_{r}^{\prime}=y_{r}+\frac{w}{2}$, where $w$ is the vehicle width. To follow the desired road, the lateral offset between the vehicle's CoG and the road centerline should be minimized. Moreover, the front and rear end of the intelligent vehicle should remain in the road region to avoid colliding with road boundaries. Moreover, the vehicle should operate safely in the road region.

\section{Parallel Steering Controller Design}

\section{A. Parallel shared steering control structure}

Considering that traffic and road conditions are constantly varying, a parallel steering control system must assist human drivers in making an intelligent vehicle complete steering tasks, ensure the safety of the intelligent vehicle, and avoid colliding with the road boundaries. This represents a multi-objective and multi-constraint optimization problem in essence. Therefore, MPC is introduced to discuss the parallel steering control issue for an intelligent vehicle. Accordingly, the structure of the parallel shared steering control employing the MPC approach is shown in Fig. 4. Here, we mainly discuss the MPC-based parallel steering control, which includes the steering relations between the human driver and the parallel steering system, a description of the vehicle's stability and collision avoidance, and weighting matrix selection based on hazard evaluation.

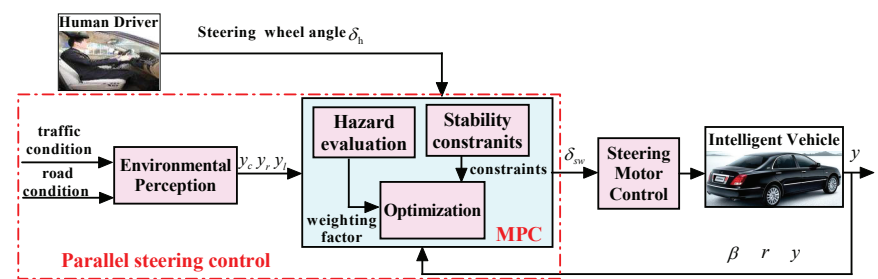

Fig. 4. Human and automatic parallel steering control structure

\section{B. Parallel Steering Controller Design Based on MPC}

1) State and output prediction for the intelligent vehicle: Because vehicle state estimation schemes have already been discussed extensively in [18], [19], it is reasonably assumed that the vehicle velocity, yaw angle, sideslip angle and tireroad friction coefficient can be estimated. Moreover, the control of the steering motor is not discussed in this manuscript.

Suppose that the predictive horizon is $p$, the control horizon is $m$, and $m \leq p$. Moreover, it is assumed that the longitudinal velocity of the intelligent vehicle keep invariant in the predictive horizon and the longitudinal velocity should be updated before next predictive horizon comes. In this situation, the variation of the longitudinal velocity is considered in the parallel steering control. In accordance with the system dynamics described in Eq. (9) and the current state of the intelligent vehicle, the dynamic behavior of the intelligent vehicle over a receding horizon is predicted as follows:

$$
\begin{aligned}
x(k+1 \mid k)= & A_{d} x(k)+B_{d} \delta_{f}(k), \\
x(k+2 \mid k)= & A_{d}^{2} x(k)+A_{d} B_{d} \delta_{f}(k)+B_{d} \delta_{f}(k+1), \\
& \vdots \\
x(k+m \mid k)= & A_{d}^{m} x(k)+A_{d}^{m-1} B_{d} \delta_{f}(k)+A_{d}^{m-2} B_{d} \delta_{f}(k+1) \\
& +\cdots+B_{d} \delta_{f}(k+m-1), \\
& \vdots \\
x(k+p \mid k)= & A_{d}^{p} x(k)+A_{d}^{p-1} B_{d} \delta_{f}(k)+A_{d}^{p-2} B_{d} \delta_{f}(k+1) \\
& +\cdots+\sum_{i=1}^{p-m+1} A_{d}^{i-1} B_{d} \delta_{f}(k+m-1) .
\end{aligned}
$$

The control input is assumed invariant when the sampling instants are beyond the control horizon $m$, that is, $u(k+m)=$ $u(k+m+1)=\cdots=u(k+p-1)$. In this situation, the output can be predicted as follows:

$$
\begin{aligned}
y(k+1 \mid k)= & C_{d} A_{d} x(k)+C_{d} B_{d} \delta_{f}(k) \\
y(k+2 \mid k)= & C_{d} A_{d}^{2} x(k)+C_{d} A_{d} B_{d} \delta_{f}(k)+C_{d} B_{d} \delta_{f}(k+1), \\
& \vdots \\
y(k+m \mid k)= & C_{d} A_{d}^{m} x(k)+C_{d} A_{d}^{m-1} B_{d} \delta_{f}(k)+ \\
& \cdots+C_{d} B_{d} \delta_{f}(k+m-1) \\
& \vdots \\
y(k+p \mid k)= & C_{d} A_{d}^{p} x(k)+C_{d} A_{d}^{p-1} B_{d} \delta_{f}(k)+ \\
& \cdots+\sum_{i=1}^{p-m+1} C_{d} A_{d}^{i-1} B_{d} \delta_{f}(k+m-1) .
\end{aligned}
$$


Define the input sequence $U(k)$ and the predicted output sequence $Y(k+1 \mid k)$ at the sampling instant $k$ as follows:

$U(k) \triangleq\left[\begin{array}{c}\delta_{f}(k) \\ \delta_{f}(k+1) \\ \vdots \\ \delta_{f}(k+m-1)\end{array}\right], Y(k+1 \mid k) \triangleq\left[\begin{array}{c}y(k+1 \mid k) \\ y(k+2 \mid k) \\ \vdots \\ y(k+p \mid k)\end{array}\right]$.

Accordingly, the $p$-step output prediction for the intelligent vehicle can be expressed as follows:

$$
Y(k+1 \mid k)=S_{x} x(k)+S_{u} U(k),
$$

where

$$
\begin{gathered}
S_{u}=\left[\begin{array}{cccc}
C_{d} B_{d} & 0 & \cdots & 0 \\
C_{d} A_{d} B_{d} & C_{d} B_{d} & \cdots & 0 \\
\vdots & \vdots & \ddots & \vdots \\
C_{d} A^{p-1} B_{d} & C_{d} A^{p-2} B_{d} & \cdots & \sum_{i=1}^{p-m+1} C_{d} A_{d}^{i-1} B_{d}
\end{array}\right] \\
S_{x}=\left[\begin{array}{llll}
C_{d} A_{d} & C_{d} A_{d}^{2} & \cdots & C_{d} A_{d}^{p}
\end{array}\right]^{T}
\end{gathered}
$$

2) Stable formulation: Considering the steady-state condition in Eq. (4), that is, $\dot{\beta}=0$, the steady-state yaw rate can be expressed as follows:

$$
m v r_{s s}=F_{y f}+F_{y r} .
$$

Assuming that the longitudinal tire forces are zero and that the effects of weight transfer are neglected, the following relationship can be obtained [20]:

$$
F_{y f, \max }+F_{y r, \max }=m g \mu,
$$

where $g$ is the gravitational constant. Combining Eq. (12) and Eq. (13), the maximum steady-state yaw rate can be obtained as follows:

$$
r_{s s, \max }=\frac{g \mu}{v}
$$

Another important consideration for vehicle lateral stability is the saturation of the tire sideslip angle. As described in Eq. (6b), the sideslip angles of the rear tires can be approximated as $\alpha_{r}=\beta-\frac{b r}{v}$ Moreover, as shown in Fig. 5, the maximum rear tire sideslip angle is $\alpha_{r, s a t}=0.15 \mathrm{rad}$, which differentiates the phase planes with unstable or stable trajectories.

Accordingly, the bound of the tire sideslip angle can be obtained as follows:

$$
\beta_{\max }=\alpha_{r, s a t}+\frac{b r}{v} .
$$

Based on the above-mentioned safety considerations, the yaw rate and sideslip angle should satisfy the following constraints:

$$
\begin{aligned}
& r_{s s, \min } \leq r \leq r_{s s, \max }, \\
& \beta_{\min } \leq \beta \leq \beta_{\max },
\end{aligned}
$$

where the minimum yaw rate and sideslip angle can be chosen as $r_{\min }=-r_{\max }$ and $\beta_{\min }=-\beta_{\max }$ [20]. Then, the following inequality can be obtained to describe stable operation for parallel steering control:

$$
C_{s} x(k+1) \leq b_{s},
$$
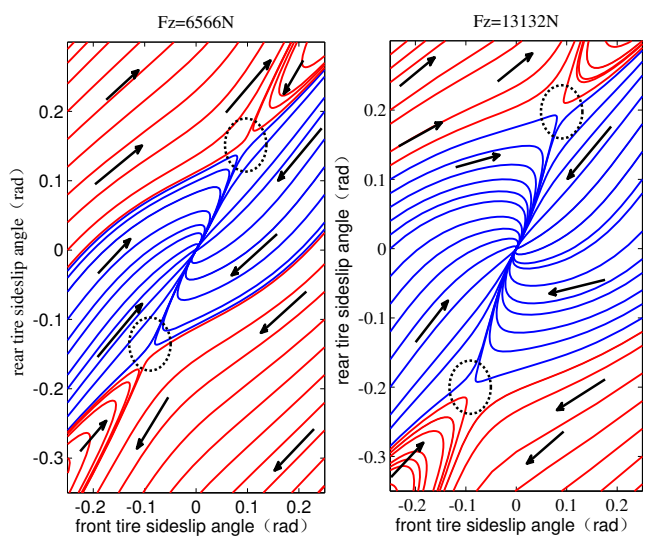

Fig. 5. Phase diagram of tire sideslip angle with different initial values of $\alpha_{f}$ and $\alpha_{r}$ under the conditions $v=18 \mathrm{~m} / \mathrm{s}$ and $\delta_{f}=0 \mathrm{rad}$.

where

$$
C_{s}=\left[\begin{array}{cccc}
0 & 0 & 0 & 1 \\
0 & 0 & 1 & -\frac{b}{v_{x}} \\
0 & 0 & 0 & -1 \\
0 & 0 & -1 & -\frac{b}{v_{x}}
\end{array}\right], b_{s}=\left[\begin{array}{c}
\frac{g \mu}{v_{x}} \\
\alpha_{r, s a t} \\
\frac{g \mu}{v_{x}} \\
\alpha_{r, s a t}
\end{array}\right] .
$$

3) Collision avoidance: To ensure that the intelligent vehicle does not collide with other vehicles, it is better to keep it running in its own lane. The above-mentioned objective can be achieved by restricting the lateral positions of the vehicle front end $F$ and the rear end $R$ within the road boundaries, that is,

$$
y_{r}^{\prime}+\omega_{p} \leq y_{i} \leq y_{l}^{\prime}-\omega_{p}, \quad i=F, R,
$$

where $\omega_{p}$ is a small positive number selected to avoid colliding with the road boundaries.

The relationship among the vehicle lateral position, front end $F$ and rear end $R$ can be described as [6]

$$
\begin{aligned}
& y_{F}=y_{o}+l_{f}(\psi+\beta), \\
& y_{R}=y_{o}-l_{r}(\psi+\beta) .
\end{aligned}
$$

where $y_{F}$ and $y_{R}$ are the lateral positions of the front end and rear end of the intelligent vehicle, respectively; $y_{o}$ is the lateral position of the CoG of the intelligent vehicle; $\psi$ is the yaw angle; and $\beta$ is the sideslip angle.

Substituting Eq. (19) into Eq. (18), collision avoidance can be achieved by restricting the lateral position of the intelligent vehicle to satisfy the following constraints:

$$
C_{r} x(k+i) \leq b_{r},
$$

where

$C_{r}=\left[\begin{array}{cccc}1 & l_{f} & l_{f} & 0 \\ -1 & -l_{r} & -l_{r} & 0 \\ 1 & -l_{r} & -l_{r} & 0 \\ -1 & l_{r} & l_{r} & 0\end{array}\right], b_{r}=\left[\begin{array}{c}y_{l}-\frac{w}{2}-\omega_{p} \\ -y_{r}-\frac{w}{2}-\omega_{p} \\ y_{l}-\frac{w}{2}-\omega_{p} \\ -y_{r}-\frac{w}{2}-\omega_{p}\end{array}\right]$.

4) Actuator saturation formulation: To avoid saturation of the mechanical system, the steering action of the steering wheel motor should be limited. The corresponding requirement is formulated as the constraints

$$
\left|\delta_{f}(k+i)\right| \leq \delta_{f, s a t},
$$


where $\delta_{f}(k+i)$ is the front steering wheel angle at time $k+i$ and $\delta_{f, s a t}$ is the maximum front steering wheel angle.

In addition, to ensure that the steering operation is smooth, the control action between two sample instants should be minimized:

$$
\left|\Delta \delta_{f}(k+i)\right| \leq \dot{\delta}_{f, s a t} T_{s} .
$$

5) Moving horizon parallel steering control: As shown in Fig. $4, \delta_{f}$ is the actual front steering wheel angle implemented for the intelligent vehicle. The steering operation by the human driver will remain valid until the driver cannot steer the intelligent vehicle to follow the desired road or makes the intelligent vehicle unstable. Therefore, the actual steering wheel angle should follow the steering wheel angle offered by the human driver, which can be described as minimizing the difference between the actual front steering wheel angle $\delta_{f}$ and the human driver steering wheel angle $\delta_{h}$ as follows:

$$
J_{1}=\left|\delta_{f}(k)-\delta_{h}(k)\right| \text {. }
$$

In addition, considering the mechanical characteristics of the steering actuator and to ensure the smooth performance of the control system, the difference between two steering actions should be limited. The corresponding requirement is described as follows:

$$
J_{2}=\sum_{i=1}^{p}\left(\Delta \delta_{f}(k+i)\right)^{2},
$$

where $\Delta \delta_{f}(k+i)=\delta_{f}(k+i)-\delta_{f}(k+i-1)$.

To minimize $J_{1}$ and $J_{2}$ simultaneously, weighting factors are introduced. Thus, following the operation of the human driver, the multi-objective cost function is defined as follows:

$$
J_{H}=\Gamma_{h}\left|\delta_{f}(k)-\delta_{h}(k)\right|+\Gamma_{d} \sum_{i=1}^{p}\left(\Delta \delta_{f}(k+i)\right)^{2},
$$

where $\Gamma_{h}$ is a positive weighting factor and $\Gamma_{d}=$ $\operatorname{diag}\left(\tau_{d, 1}, \tau_{d, 2}, \cdots, \tau_{d, p}\right)$ is the weighting matrix. The objective function $J_{H}$ describes the issue whereby the parallel steering control action should follow the operation of the human driver as much as possible.

Considering the fact that the intelligent vehicle will deviate from the road centerline or experience instabilities resulting from improper operation by the human driver, it is necessary to consider the road-following and stability performance of parallel steering control. Accordingly, an additional objective function is introduced as follows:

$$
J_{A}=\Gamma_{y}\left\|Y(k+1)-Y_{c}(k+1)\right\|+\Gamma_{\beta}\|(\beta(k+i))\|,
$$

where the first element $\left\|Y(k+1)-Y_{c}(k+1)\right\|$ of $J_{A}$ is assumed as following the road centerline and the second element $\|(\beta(k+i))\|$ is considered as the stability of the intelligen$\mathrm{t}$ vehicle. Moreover, $\Gamma_{y}=\operatorname{diag}\left(\Gamma_{y, 1}, \Gamma_{y, 2}, \cdots, \Gamma_{y, p}\right)$ and $\Gamma_{\beta}=\operatorname{diag}\left(\Gamma_{\beta, 1}, \Gamma_{\beta, 2}, \cdots, \Gamma_{\beta, p}\right)$ are the weighting matrices.

From Eq. (26), the autonomous driving condition is considered in the objective function of the parallel steering control to make the intelligent vehicle follow the road centerline and ensure stability. To minimize both $J_{H}$ and $J_{A}$, a weighting matrix is introduced, and the objective of the parallel steering control can be described as follows:

$$
J(y(k), U(k), m, p)=J_{H}+\Gamma J_{A},
$$

where $\Gamma$ is the weighting factor.

According to the objective function defined in Eq. (27) and the constraints described in Eq. (17), Eq. (20), Eq. (21) and Eq. (22), the MPC-based optimization problem for parallel steering control can be defined as follows:

$$
\begin{array}{ll} 
& \min _{U(k)} E q .(27) \\
\text { s.t. } \quad & x(k+1)=A_{d} x(k)+B_{d} \delta_{f}(k), \\
& y(k+1)=C_{d} x(k), \\
& x(k \mid k)=x(k), \\
& C_{r} x(k+i) \leq b_{r} \\
& C_{s} x(k+i) \leq b_{s} \\
& i=1,2, \cdots, p \\
& \left|\delta_{f}(k+j)\right| \leq \delta_{f, \text { sat }} \\
& \left|\Delta \delta_{f}(k+j)\right| \leq \dot{\delta}_{f, \text { sat }} T_{s}, \\
& j=1,2, \cdots, m,
\end{array}
$$

At each execution of the controller, the optimization problem defined in Eq. (28) is solved for each sample instant, and the optimal input corresponding to the lowest objective value is used. As is common with MPC, the first element of the optimal control sequence is applied to the intelligent vehicle, and the optimization problem is re-solved in the next sample instant. Thus, parallel steering control by a human driver and autonomous driving system can be achieved.

The difference between the scheme in [6] and the approach in this manuscript is that the control issue is differentiated from path following and parallel steering control. Despite MPC control approach is used in these two control issues, the cost functions are selected different and the safety constraints are additional considered in the proposed scheme here.

\section{Weighting Factor Selection Based on Hazard Evaluation}

It is concluded from Eq. (27) that the weighting factor $\Gamma$ regulates the weights between the human driver and the autonomous steering system. Therefore, the choice of the weighting factor $\Gamma$ is critical. In this manuscript, the weighting factor is selected based on road and steering operation hazard evaluation.

The road hazard is employed to describe the vehicle position dangerous for the intelligent vehicle as follows:

$$
E_{\text {road }}=\left(y(k)-y_{c}(k)\right)^{E_{A}},
$$

where $E_{A}>0$ is a positive constant.

The road hazard is not sufficient enough to balance the weights of the driver and the auto-controller without knowing the information of the current driver's status. Several aspects should be considered when chooses the parameter to reflect the current driver's behavior. The first one is that the parameter should be selected to reflect the performance of driver's behavior. The second is that the adopted system's information 
under the current scheme should not introduce additional sensor information. The third is that the information from the parallel steering controller should be sufficient considered and used.

$$
E_{\text {driver }}=\frac{\left|\delta_{\text {human }}(k)-\delta_{f}(k \mid k-1)\right|}{E_{B}},
$$

where $E_{B}$ is a regulating factor that makes $E_{\text {road }}, E_{\text {driver }}$ of the same order of magnitude, and $\delta(k \mid k-1)$ is the optimized front steering wheel angle at the sample time $k-1$ for current sample time.

The relationship of the weighting factor $\Gamma$, road hazard $E_{\text {road }}$ and human driver steering operation hazard $E_{\text {driver }}$ is described as a two-dimensional map, for which fuzzy logic is employed. Table I presents the inference rules between the road hazard and the human driver steering operation hazard. Fig. 6 (a)-(b) show the triangular fuzzy membership functions of the road hazard and human driver steering operation hazard which are safe (S), medium safe (MS), medium (M), medium dangerous (MD) and dangerous (D). Fig. 6 (c) show the triangular fuzzy membership functions of the the weighting factor which are small (S), medium small (MS), medium (M), medium large (ML) and large (L). Accordingly, the twodimensional map is obtained and shown in Fig. 7. Thus, the weighting factor can be obtained when the road hazard and human driver steering operation hazard are obtained.

TABLE I

INFERENCE RULES FOR WEIGHTING MATRIX USING ROAD AND HUMAN DRIVING STEERING HAZARDS

\begin{tabular}{llllll}
\hline \multirow{2}{*}{$E_{\text {driver }}$} & \multicolumn{5}{c}{$E_{\text {road }}$} \\
\cline { 2 - 6 } & $\mathrm{S}$ & $\mathrm{MS}$ & $\mathrm{M}$ & $\mathrm{MD}$ & $\mathrm{D}$ \\
\hline $\mathrm{S}$ & $\mathrm{S}$ & $\mathrm{S}$ & $\mathrm{S}$ & $\mathrm{S}$ & $\mathrm{MS}$ \\
$\mathrm{MS}$ & $\mathrm{S}$ & $\mathrm{S}$ & $\mathrm{S}$ & $\mathrm{MS}$ & $\mathrm{M}$ \\
$\mathrm{M}$ & $\mathrm{S}$ & $\mathrm{S}$ & $\mathrm{MS}$ & $\mathrm{M}$ & $\mathrm{ML}$ \\
MD & $\mathrm{S}$ & $\mathrm{MS}$ & $\mathrm{M}$ & $\mathrm{ML}$ & $\mathrm{L}$ \\
$\mathrm{D}$ & $\mathrm{MS}$ & $\mathrm{MS}$ & $\mathrm{M}$ & $\mathrm{ML}$ & $\mathrm{L}$ \\
\hline
\end{tabular}

\section{RESULTS AND DiscusSiON}

In this section, the Hongqi intelligent vehicle is built to verify the proposed parallel steering controller. Then, the joint Simulink-veDYNA simulations are performed to determine the effectiveness of the proposed hazard-evaluation-oriented parallel steering controller.

\section{A. Vehicle Model for Control Performance Verification}

To investigate the performance of the proposed hazardevaluation-oriented moving horizon parallel steering control scheme for an intelligent vehicle, the versatile vehicle dynamics simulation software veDYNA ${ }^{\circledR}$ is employed, and joint Simulink-veDYNA ${ }^{\circledR}$ simulations have been conducted. veDYNA contains a modular model architecture implemented in MATLAB, and the driver model, which is determined based on the driving style and driving skill, is included in the software. The driver model contained in veDYNA is similar with the model presented in [21]. Therefore, the driver operation can be fully considered in the parallel steering control.

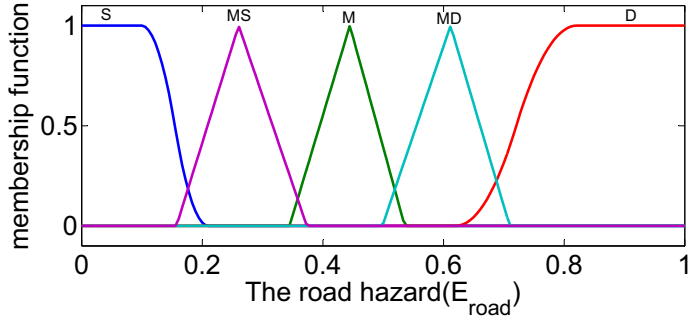

(a) triangle membership function of road hazard

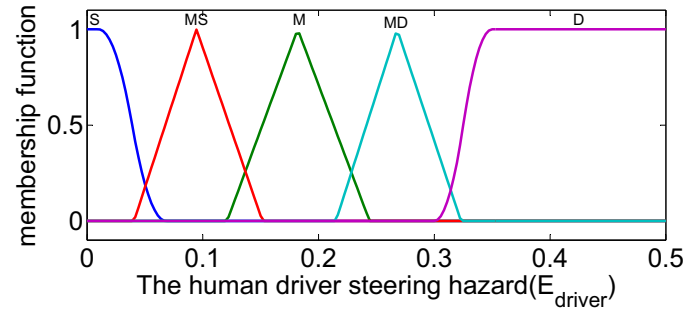

(b) Triangular membership function of human driver hazard

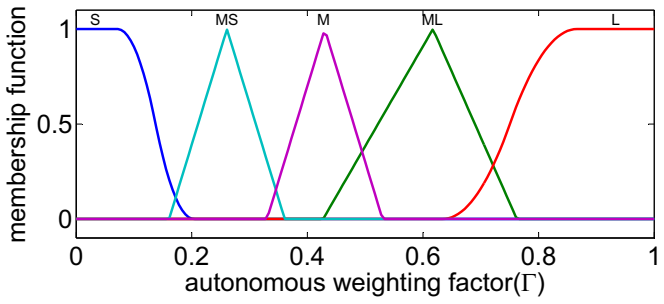

(c) weighting factor

Fig. 6. membership function for road hazard, human driver hazard and weighting factor

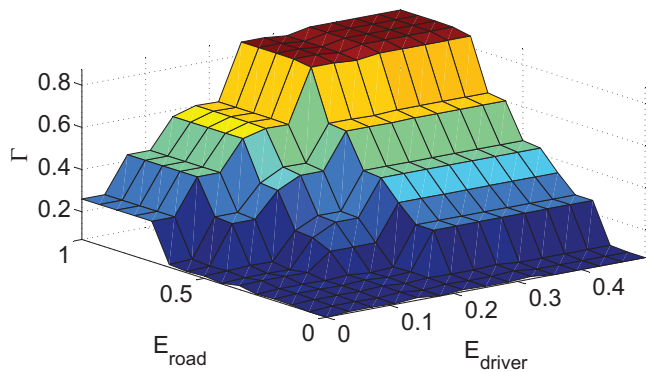

Fig. 7. Weighting factor based on hazard evaluation

To reflect the dynamic characteristics of an intelligent vehicle, a highly efficient vehicle model that can reflect most of the vehicle dynamics under normal and critical operating conditions is needed. Therefore, an intelligent vehicle model of a Hongqi HQ430 passenger car shown in Fig. 8(a) is utilized to be co-simulated and to verify the effectiveness of the controller proposed in this manuscript. The detailed model structure of the Hongqi HQ430 intelligent vehicle can be seen in Fig. 8(b); the vehicle is established and extended based on the vehicle dynamics software veDYNA. The basic and empirical parameters of the HQ430 can be obtained from its technical documentation. The characteristic parameters of the HQ430 can be identified from experimental data obtainable from various types of characteristic experiments. Moreover, because veDYNA does not contain an automatic transmission and because an A761E automatic transmission was equipped 
on the Hongqi HQ430, it is essential to discuss the modeling of the transmission to improve the accuracy of the Hongqi HQ430 intelligent vehicle model.

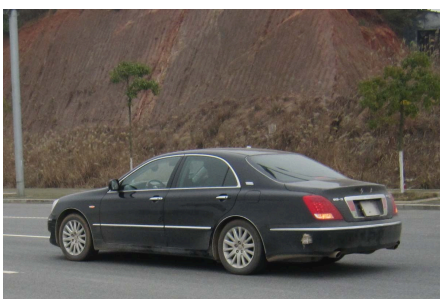

(a) Actual picture of Hongqi HQ430 vehicle

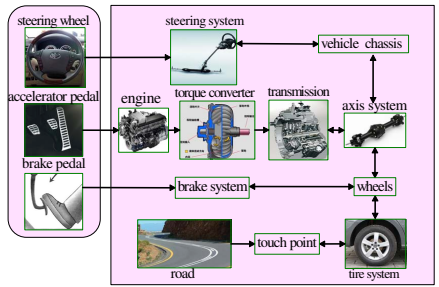

(b) HQ430 simulation model structure

Fig. 8. Actual picture and simulation model of Hongqi HQ430 vehicle

Based on the above model and the identified parameters of the Hongqi HQ430 passenger vehicle, the longitudinal and lateral dynamics are both validated through representative vehicle experiments. Accordingly, the average longitudinal dynamic precision is $91.3 \%$, and the average lateral precision can be obtained as $79.4 \%$. The detailed verification is introduced in [22].

\section{B. Simulation Results}

According to the hazard-evaluation-oriented parallel steering control issue described by Eq. (28), the predictive horizon and the control horizon are selected as $m=25$ and $P=25$, respectively. Then, the weighting factor $\Gamma_{h}=100$ is chosen to track the operation of the human driver. The weighting matrix $\Gamma_{d}=\operatorname{diag} 1,1, \cdots, 1$ is selected to ensure that the control action is not excessive. Moreover, the weighting matrices $\Gamma_{y}=\operatorname{diag}(1,1, \cdots, 1)$ and $\Gamma_{\beta}=\operatorname{diag}(100,100, \cdots, 100)$, for the purpose of automated driving, make the actual lateral position of the intelligent vehicle follow the lateral position of the road centerline and ensure the stability of the intelligent vehicle, respectively.

1) Safe driving verification: To verify the effectiveness of the proposed hazard-evaluation-oriented parallel steering control in safe driving situations, the simulation verification is performed under the condition of a slalom maneuver on an asphalt road with a friction coefficient of $\mu=0.85$. In this scenario, the intelligent vehicle accelerates from rest to a speed of $50 \mathrm{~km} / \mathrm{h}$. Then, the slalom maneuver is performed, and the parallel steering control is implemented during the steering operation. Moreover, the proposed parallel steering control scheme is compared with another parallel steering control scheme that did not consider the hazard evaluation whereby the objective function is $J(y(k), U(k), m, p)=J_{H}$ only and the other constraints and initial conditions are the same as in the optimization in Eq. (28). The simulation results for this scenario are shown in Fig. 9 (a)-(d).

It can be seen from Fig. 9 (a) and (b) that the yaw rate and sideslip angle are small, which indicates that the intelligent vehicle is operating safely during the slalom maneuver. Because the human driver can operate the intelligent vehicle and complete this operating task at a relatively low velocity, the parallel steering control scheme only needs to follow the operation of the human driver and ensure that the difference between two steering actions is not excessive, which can be described by $J(y(k), U(k), m, p)=J_{H}$. Therefore, it can be concluded that the proposed hazard-evaluation-oriented parallel steering control scheme is similar to the parallel steering control scheme, which does not consider the hazard evaluation situation under safe driving conditions. The conclusion can also be verified from Fig. 9 (c) that the actual front steering angle acting on the intelligent vehicle obtains a very small error considering operation that does not consider the hazard evaluation. Moreover, the lateral position obtained from the parallel steering control scheme is the same as that in the scheme that does not consider the hazard evaluation. These situations specify that the proposed hazard-evaluation-oriented parallel steering control scheme is similar to the scheme without considering the hazard evaluation when the intelligent vehicle operates in a safe manner by a driver. It also verifies that the control logic of the hazard-evaluation-oriented parallel steering control is correct.
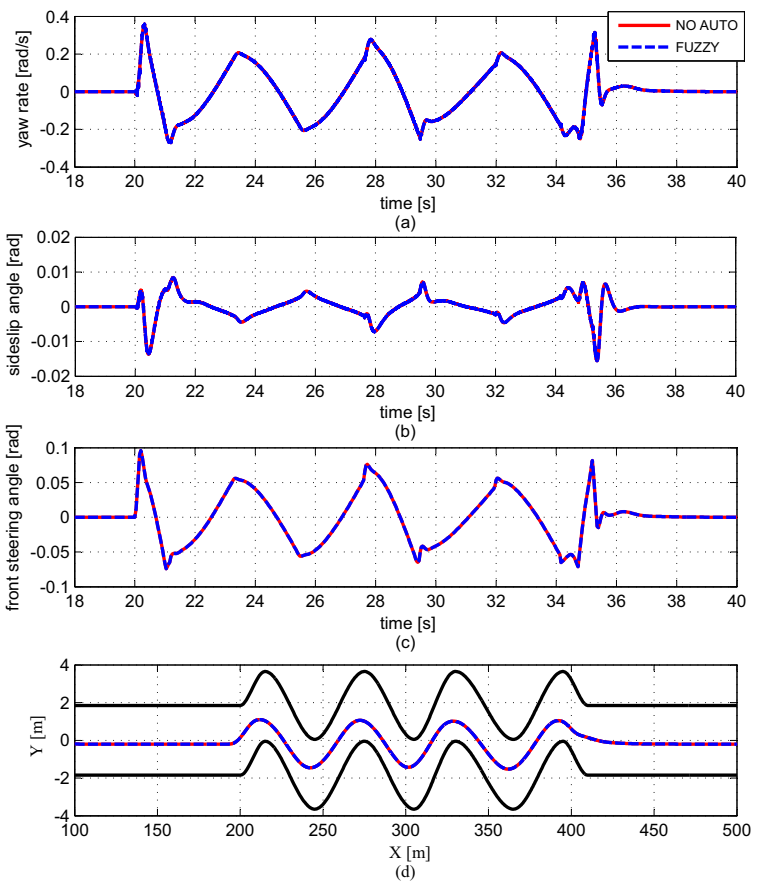

Fig. 9. Simulation results for parallel steering control under slalom maneuver. "NO AUTO" denotes parallel steering control without considering the roadfollowing and stability performance $J_{A}$, and "FUZZY" denotes the proposed hazard-evaluation-oriented parallel steering control approach.

2) Hazardous driving verification: To validate the performance of the proposed parallel steering control approach under hazardous operating conditions, a double-lane-change 
maneuver is performed on a wet asphalt road with a friction coefficient of $\mu=0.6$. Under this operating condition, the velocity of the intelligent vehicle is $100 \mathrm{~km} / \mathrm{h}$. It is hazardous for the intelligent vehicle to perform this operation while operated by a human driver directly. To demonstrate the effectiveness of the proposed hazard-evaluation-oriented parallel steering control approach, we compare it with the parallel steering control scheme that did not consider the hazard evaluation; the comparison results are shown in Fig. 10 (a)-(d).

It can be observed from the sideslip angle shown in Fig. 10 (a) that the maximum sideslip angle is larger than $0.1 \mathrm{rad}$. This indicates that the intelligent vehicle tended to lose stability. Moreover, it can be concluded from Fig. 10 (a) that the sideslip angle obtained from the approach in this manuscript is smaller than that in the scheme that did not consider the hazard evaluation. The result indicates that the proposed hazard-evaluationoriented parallel steering control scheme is more effective than when not considering the hazard evaluation approach. The conclusion can also be obtained from the yaw rate shown in Fig. 10 (b). The intelligent vehicle can perform the doublelane-change maneuver under the control of these two parallel steering control schemes, as can be seen from Fig. 10 (c). This indicates that these two parallel steering control approaches can regulate steering operations implemented by a human driver. Moreover, compared with the parallel steering control scheme that did not consider the hazard evaluation, the parallel steering control approach introduced in this manuscript obtains better performance, as shown in Fig. 10 (c), by regulating the front steering wheel angle shown in Fig. 10 (d). This is because the hazard evaluation plays an active role in the hazardous situation, thereby obtaining better performance. In addition, the road hazard factor $E_{\text {road }}$ and the human driver steering hazard factor $E_{\text {driver }}$ for these two schemes are compared, and the results are shown in Fig. 10 (e) and (f), respectively. The human driver steering hazard factor obtained from the approach in this manuscript is substantially smaller than that in the approach not considering the hazard evaluation. The road hazards under these two methods are similar and only present small differences at approximately 22-23 seconds. This is because these two methods obtain similar path-tracking performances, as shown in Fig. 10 (c), while the front steering wheel angle is different, as shown in Fig. 10 (d). It can also be concluded from the comparison results that the hazardevaluation-oriented parallel steering control is more effective than when not considering the hazard evaluation approach because the human driver steering hazard is smaller.

3) Improper operation by driver: We further validate the performance of the proposed hazard-evaluation-oriented parallel steering control scheme for the situation of improper operation by the human driver. The verification results are shown in Fig. 11 (a)-(d).

It can be concluded from the trajectory of the intelligent vehicle shown in Fig. 11 (a) that the human driver operates the intelligent vehicle beyond the driving lane in this situation. In this case, both the parallel steering control scheme proposed in this manuscript and the approach that does not consider the hazard evaluation can regulate the front steering wheel angle, as shown in Fig. 11 (b). This is because the constraints


Fig. 10. Simulation results for parallel steering control under the doublelane-change maneuver. "NO AUTO" denotes parallel steering control without considering the road-following and stability performance $J_{A}$, and "FUZZY" denotes the proposed hazard-evaluation-oriented parallel steering control approach.

play an effective role and restrict the lateral movement of the intelligent vehicle. Moreover, because the hazard-evaluationoriented parallel steering control scheme also considers the road centerline tracking and stability performance in the objective function described in Eq. (28), the approach proposed in this manuscript is more effective than when not considering the hazard evaluation, which can be seen from the sideslip angle and yaw rate shown in Fig. 11 (c) and (d), respectively.

\section{CONCLUSION}

In this paper, a parallel steering control framework is proposed for an intelligent vehicle using a moving horizon optimization approach that considers the lateral stability, collision avoidance and actuator saturation sufficiently and describes them as constraints. Hazard evaluation is performed to ensure the safe operation of the intelligent vehicle based on the road hazard and the steering operation error. The intelligent vehicle will be mainly operated by the human driver when the vehicle is operated in a safe and stable manner. The automated steering driving objective will play an active role and regulate the steering operation applied to the intelligent vehicle based on the hazard evaluation. The proposed hazardevaluation-oriented moving horizon parallel steering control has been confirmed to be an effective approach in humanmachine cooperation under both conventional conditions and 

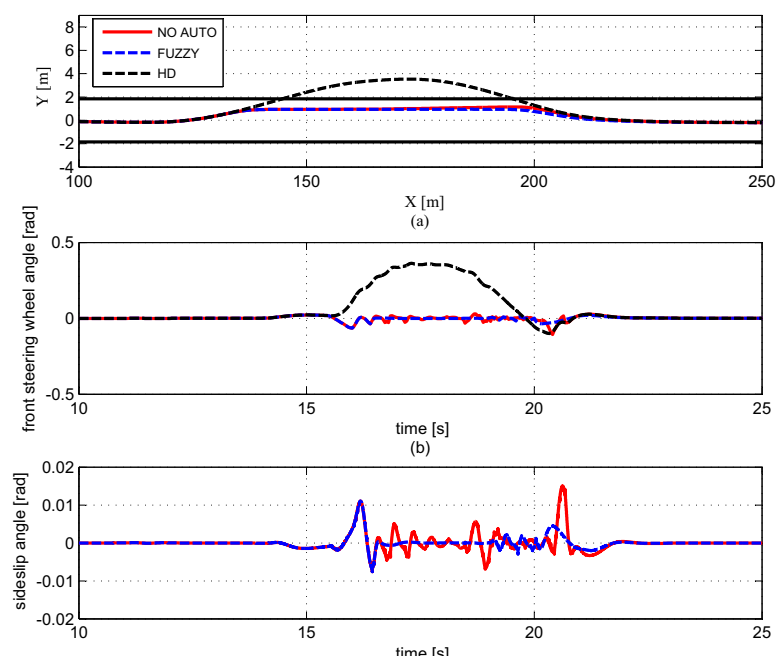

$\underset{(c)}{\operatorname{time}}[\mathrm{s}]$

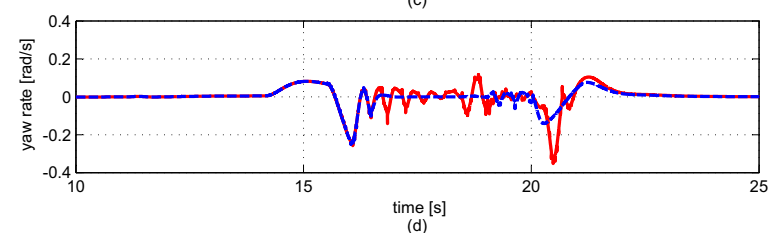

(d)

Fig. 11. Simulation results for parallel steering control considering improper operation by the human driver. "NO AUTO" denotes parallel steering control without considering the road-following and stability performance $J_{A}$, "FUZZY" denotes the proposed hazard-evaluation-oriented parallel steering control approach, and "HD" denotes the operation by the human driver.

hazardous conditions.

In the future works, considering the current steering difference between human driver and actual front steering wheel angle may cause some drastic switch jitter which may cause driver more nervous, the minimization problem of Eq. (23) should be considered as $J_{1}=\sum_{i=k-p}^{k}\left|\delta_{f}(i)-\delta_{h}(i)\right|$ since the future operation of human driver cannot be obtained. Moreover, the similar situation should be discussed in Eq. (30).

\section{REFERENCES}

[1] F.-Y. Wang, N.-N. Zheng, D. P. Cao, C. M. Martinez, L. Li, and T. Liu, "Parallel driving in CPSS: a unified approach for transport automation and vehicle intelligence," IEEE/CAA Journal of Automatica Sinica, vol. 4, no. 4, pp. 577-587, 2017.

[2] F.-Y. Wang, J. Zhang, Q. L. Wei, X. H. Zheng, and L. Li, "PDP: parallel dynamic programming," IEEE/CAA of Automatica Sinca, vol. 4, no. 1, pp. 1-5, 2017.

[3] T. X. Bai, S. Wang, Z. Shen, D. P. Cao, N. N. Zheng, and F. Y. Wang, "Parallel robotics and parallel unmanned systems: Framework, structure, process, platform and applications," Acta Automatica Sinica, vol. 43, no. 2, pp. 161-175, 2017.

[4] F. Y. Wang, "Scanning the issue and beyond: Parallel driving with software vehicular robots for safety and smartness," IEEE Transactions on Intelligent Transportation System, vol. 15, no. 4, pp. 1381-1387, Aug. 2014.

[5] F. Naser, D. Dorhout, S. Proulx, S. D. Pendleton, H. Andersen, W. Schwarting, L. Paull, J. Alonso-Mora, M. H. Ang Jr., S. Karaman, R. Tedrake, J. Leonard, and D. Rus, "A parallel autonomy research platform," in In Proceedings of 2017 IEEE Intelligent Vehicles Symposium (IV). USA: IEEE, 2017, pp. 933-940.

[6] H. Y. Guo, J. Liu, D. P. Cao, H. Chen, R. Yu, and C. Lv, "Dual-enveloporiented moving horizon path tracking control for fully automated vehicles," Mechatronics, Mar. online.
[7] S. J. Anderson, S. B. Karumanchi, K. Iagnemma, and J. M. Walker, "The intelligent copilot: a constraint-based approach to shared-adaptive control of ground vehicles," IEEE Intelligent Transportation Systems Magazine, vol. 5, no. 2, pp. 45-54, 2013.

[8] F.-Y. Wang, X. Wang, L. X. Li, and L. Li, "Control 5.0: from newton to merton in popper's cyber-social-physical spaces," IEEE/CAA of Automatica Sinca, vol. 34, no. 4, pp. 223-234, 2016.

[9] A. T. Nguyen, C. Sentouh, and J. C. Popieul, "Driver-automation cooperative approach for shared steering control under multiple system constraints: design and experiments," IEEE Transactions on Industrial Electronics, vol. 64, no. 5, pp. 3819-3830, 2017.

[10] F.-Y. Wang, "Parallel control and management for intelligent transportation systems: concepts, architectures, and applications," IEEE Transaction on Intelligent Transportation System, vol. 11, no. 3, pp. 630-638, 2010.

[11] A. Balachandran, S. M. Brown, M. Erlien, and J. C. Gerdes, "Predictive haptic feedback for obstacle avoidance based on model predictive control," IEEE Transactions on Automation Science and Engineering, vol. 13, no. 1, pp. 26-31, 2016.

[12] T. Kawabe, H. Nishira, and T. Ohtsuka, "An optimal path generator using a receding horizon control scheme for intelligent automobiles," in Proceedings of the 2004 IEEE International Conference on Control Applications. Taipei, Twaiwan: IEEE, 2004, pp. 1597-1602.

[13] S. Glaser, B. Vanholme, and S. Mammar, "Maneuver-based trajectory planning for highly autonomous vehicles on real road with traffic and driver interaction," IEEE Transaction on Intllignet Transportation Systmes, vol. 11, no. 3, pp. 589-606, 2010.

[14] N. M. Enache, S. Mammar, M. Netto, and B. Lusetti, "Driver steering assistance for lane-departure avoidance based on hybrid automata and composite lyapunov function," IEEE Transaction on Intelligent Transportation Systems, vol. 11, no. 1, pp. 28-39, 2010.

[15] L. Saleh, P. Chevrel, F. Claveau, J.-F. Lafay, and F. Mars, "Shared steering control between a driver and an automation: stability in the presence of driver behavior uncertainty," IEEE Transaction on Intelligent Transprotation System, vol. 14, no. 2, pp. 974-983, 2013.

[16] S. M. Erlien, S. Fujita, and J. C. Gerdes, "Shared steering control using safe envelopes for obstacle avoidance and vehicle stability," IEEE Transaction on Intelligent Transportation Systems, vol. 17, no. 2, pp. 441-451, 2016

[17] H. Dahmani, M. Chadli, A. Rabhi, and A. Hajjaji, "Road curvature estimation for vehicle lane departure detection using a robust TakagiSugeno fuzzy observer," Vehicle Systme Dynamics, vol. 51, no. 5, pp. 581-599, 2013.

[18] H. Y. Guo, H. Chen, F. Xu, F. Wang, and G. L. Lu, "Implementaiton of EKF for vehicle velocities estimation on FPGA," IEEE Transactions on Industrial Electronics, vol. 60, no. 9, pp. 3823 - 3835, 2013.

[19] H. Y. Guo, H. Chen, D. P. Cao, and W. W. Jin, "Design of a reducedorder non-linear observer for vehicle velocities estimation," IET Control Theory and Applications, vol. 7, no. 17, pp. 2056-2068, 2013.

[20] C. E. Beal and J. C. Gerdes, "Model predictive control for vehicle stabilization at the limits of handling," IEEE Transaction on Control Systems Technology, vol. 21, no. 4, pp. 1258-1269, 2013.

[21] H. T. Ding, K. H. Guo, F. Wan, J. Y. Cao, and Y. Tian, "An analytical driver model for arbitrary path following at varying vehicle speed," International Journal Vehicle Autonomous System, vol. 5, no. 3/4, p. $204-218,2007$.

[22] H. Y. Guo, F. Liu, F. Xu, H. Chen, and Y. Ji, "Nonlinear model predictive lateral stability control of active chassis for intelligent vehicles and its FPGA implementation," IEEE Transactions on Systems, Man, and Cybernetics: Systems, Mar. to be published.

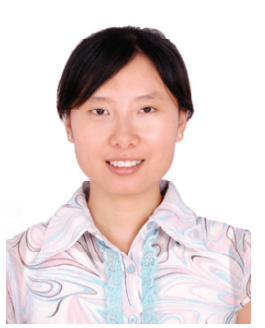

Hongyan Guo (M'17) received the Ph.D. degree from the Jilin University, Changchun, China, in 2010. She joined Jilin University, Changchun, China, in 2011. From 2014, she is a associate professor with the Department of Control Science and Engineering, Jilin University. Her current research interests include path tracking and stability control of autonomous vehicles and vehicle states estimation. 


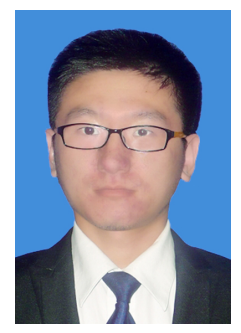

Linhuan Song received the B.Sc. degree in automation from Jilin University, Changchun, China, in 2015, where he is currently pursuing the M.Sc. degree with the Department of Control Engineering and Control Theory. His current research interests include active safety and intelligent vehicle, particularly driver-automation cooperation steering of intelligent vehicle.

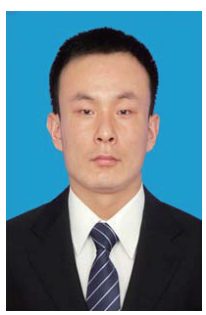

Jun Liu received the M. S and B.Sc. degree in automation from Jilin University, Changchun, China, in 2014, where he is currently pursuing the D.Sc. degree with the Department of Control Engineering and Control theory. His current research interests include vehicle stability control, autonomous driving, and human-vehicle cooperative systems.

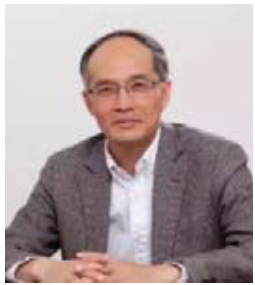

Feiyue Wang (S'87-M'89-SM'94-F'03) received his $\mathrm{Ph} . \mathrm{D}$. in Computer and Systems Engineering from Rensselaer Polytechnic Institute, Troy, New York in 1990. He joined the University of Arizona in 1990 and became a Professor and Director of the Robotics and Automation Lab (RAL) and Program in Advanced Research for Complex Systems (PARCS). In 1999, he founded the Intelligent Control and Systems Engineering Center at the Institute of Automation, Chinese Academy of Sciences (CAS), Beijing, China, under the support of the Outstanding Overseas Chinese Talents Program from the State Planning Council and "100Talent Program" from CAS, and in 2002, was appointed as the Director of the Key Lab of Complex Systems and Intelligence Science, CAS. From 2006 to 2010, he was Vice President for Research, Education, and Academic Exchanges at the Institute of Automation, CAS. In 2011, he became the State Specially Appointed Expert and the Director of the State Key Laboratory of Management and Control for Complex Systems. Dr. Wang's current research focuses on methods and applications for parallel systems, social computing, and knowledge automation. He was the Founding Editor-in-Chief of the International Journal of Intelligent Control and Systems (1995-2000), Founding EiC of IEEE ITS Magazine (2006-2007), EiC of IEEE Intelligent Systems (2009-2012), and EiC of IEEE Transactions on ITS (2009-2016). Currently he is EiC of IEEE Transactions on Computational Social Systems, Founding EiC of IEEE/CAA Journal of Automatica Sinica, and Chinese Journal of Command and Control. Since 1997, he has served as General or Program Chair of more than 20 IEEE, INFORMS, ACM, and ASME conferences. He was the President of IEEE ITS Society (2005-2007), Chinese Association for Science and Technology (CAST, USA) in 2005, the American Zhu Kezhen Education Foundation (2007-2008), and the Vice President of the ACM China Council (2010-2011). Since 2008, he has been the Vice President and Secretary General of Chinese Association of Automation. Dr. Wang has been elected as Fellow of IEEE, INCOSE, IFAC, ASME, and AAAS. In 2007, he received the National Prize in Natural Sciences of China and was awarded the Outstanding Scientist by ACM for his research contributions in intelligent control and social computing. He received IEEE ITS Outstanding Application and Research Awards in 2009, 2011 and 2015, and IEEE SMC Norbert Wiener Award in 2014.

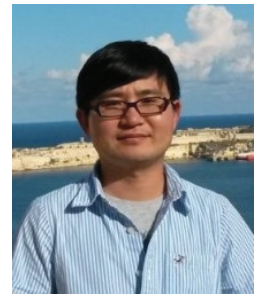

Dongpu Cao (M'08) received the Ph.D. degree from Concordia University, Canada, in 2008. He is currently an Associate Professor at University of Waterloo, Canada. His research focuses on vehicle control and intelligence, automated driving and parallel driving, where he has contributed more than 130 publications and 1 US patent. He received the ASME AVTT' 2010 Best Paper Award and 2012 SAE Arch T. Colwell Merit Award. Dr. Cao serves as an Associate Editor for IEEE TRANSACTIONS ON VEHICULAR TECHNOLOGY, IEEE TRANSACTIONS ON INTELLIGENT TRANSPORTATION SYSTEMS, IEEE/ASME TRANSACTIONS ON MECHATRONICS, IEEE TRANSACTIONS ON INDUSTRIAL ELECTRONICS and ASME JOURNAL OF DYNAMIC SYSTEMS, MEASUREMENT AND CONTROL. He has been a Guest Editor for VEHICLE SYSTEM DYNAMICS, and IEEE TRANSACTIONS ON HUMAN-MACHINE SYSTEMS. He has been serving on the SAE International Vehicle Dynamics Standards Committee and a few ASME, SAE, IEEE technical committees.

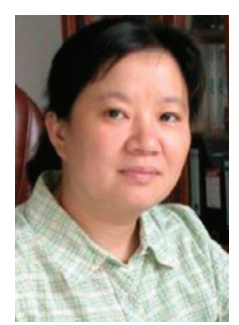

Hong Chen (M'02-SM'12)received the Ph.D. degree from the University of Stuttgart, Stuttgart, Germany, in 1997. She joined Jilin University of Technology, Changchun, China, in 1986, where she became an Associate Professor in 1998 and has been a Professor since 1999. Her current research interests include model predictive control, optimal and robust control, and applications in process engineering and mechatronic systems.

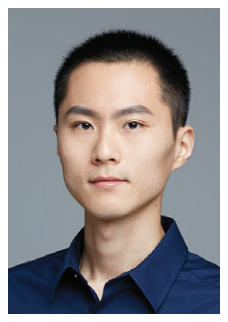

Chen Lv is currently a research fellow at Advanced Vehicle Engineering Center, Cranfield University, UK. He received the Ph.D. degree at Department of Automotive Engineering, Tsinghua University, China in 2016. From 2014 to 2015, he was a joint PhD researcher at EECS Dept., University of California, Berkeley, supervised by Prof. Alberto L. Sangiovanni-Vincentelli. His research focuses on cyber-physical system, hybrid system, advanced vehicle control and intelligence, where he has contributed over 40 papers and obtained 10 Chinese

patents..

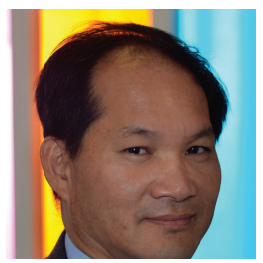

Patrick Chi-Kwong LUK (M’95 - SM’10) was born in Hong Kong. He received the High Diploma (BSc) from Hong Kong Polytechnic University (PolyU), in 1983, the M.Phil degree from Sheffield University, U.K., in 1989, and the Ph.D. degree from the University of South Wales, U.K., in 1992, all in electrical engineering. He started his career in industry as Engineer Trainee between 1981 and 1983 at GEC (H.K.) and then after graduation as Applications Engineer at Polytek Engineering Co. (H.K.). In 1986, he worked as Senior Researcher in the Industrial Centre at PolyU. Since 1988, he had held academic positions at the University of South Wales, Robert Gordon University, U.K., and the University of Hertfordshire, U.K. He joined Cranfield University, U.K., in 2002, where he is a Chair Professor in electrical engineering. He was a visiting professor at Shanghai Jiaotong University in 2014. He has authored over 200 publications in electric machines and power electronics. His current research interests include electrification in transport systems and energy conversions. 